\title{
Echo chambers, confirmation bias e polarizzazione delle idee: come viaggia la conoscenza, scientifica e non, nel web 2.0.
}

\author{
Maria Rinaldi Miliani ${ }^{1}$, Giuseppe Quintaliani ${ }^{2}$ \\ ${ }^{1}$ Copywriter e consulente Social Media Strategy per la comunicazione in sanità, Perugia \\ ${ }^{2}$ Nefrologo, Segretario Fondazione Italiana del rene, Editor Renalgate.it, Perugia
}

\begin{abstract}
Echo chambers, confirmation bias and polarization of ideas: how scientific and non-scientific knowledge spreads around the Web 2.0

The internet, whether one likes it or not, has become one of the principal means of access to scientific and nonscientific knowledge. The mechanisms regulating the dissemination of facts and information online are complex and partially unknown. For some of them, such as confirmation bias and polarization, specific mathematical models have been provided. Although a lot of work still needs to be done, it is already possible to develop strategic behaviors aimed at countering disinformation. Anyone who works in the medical field or in science in general must not ignore these themes, because they are likely to become the foundation of future health communication and reliable scientific knowledge distribution online.
\end{abstract}

Keywords: Confirmation bias, Polarization, Echo chambers, e-Health

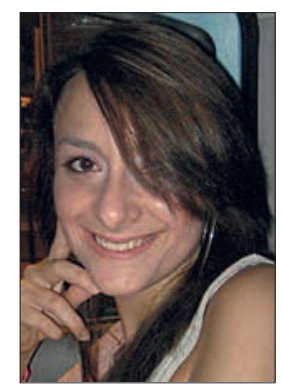

Maria Rinaldi Miliani

\section{Introduzione}

Il mondo dell'informazione da sempre è afflitto da una piaga che pare invincibile; la bufala (1). Le notizie false, soprattutto se si parla di scienza e medicina, sono sempre esistite e non è nella tecnologia che si può individuare la causa della loro nascita e diffusione (2).

Il tanto demonizzato web, al massimo, ha peggiorato la situazione, grazie alla sua intrinseca rapidità e capacità di immagazzinamento di nozioni, idee e documenti (3).

La rete ha raggiunto una pervasività inimmaginabile fino $a$ pochissimi anni fa, la quale ha portato, come afferma Luca De Biase nel suo Facts and cats. For State of the net, a "diffidare

Accepted: November 10, 2017

Published online: November 21, 2017

Indirizzo per la corrispondenza:

Dr. Giuseppe Quintaliani

Via Maturanzio, 31

06124, Perugia

quintaliani@yahoo.it maggiormente dei fatti", per tre ragioni:

1. Perché sono troppi.

2. Perché è difficile distinguere tra verità e falsi.

3. Perché sono sempre meno utili in una società nella quale le persone tendono a interpretare gli avvenimenti in maniera soggettiva.

\section{E perché si interpreta con tanta facilità?}

In un mondo come quello online, per ora non vi è traccia di dimensioni fisiche quali spazio (manca la territorialità) e tempo. Quindi, gli unici punti fissi ai quali affidarsi per percepire identità e appartenenza (due dimensioni dell' lo essenziali nella psiche umana) sono le idee personali e le informazioni (4).

Questo strapotere della soggettività è alla base della nuova comunicazione digitale e anche della divulgazione scientifica e medica moderna, fake-news comprese.

II dilagare di informazioni false online è uno dei temi caldi del momento, che colpisce la sensibilità soprattutto di medici, ricercatori e scienziati di vario genere.

Spesso ci si chiede cosa abbia portato a una deriva così incontrollata delle falsità o presunte tali, senza tenere in considerazione due ingranaggi fondamentali dell'orologio internet: la psicologia umana, individuale e collettiva, e la strutturazione originale e originaria del web.

Per quanto riguarda quest'ultimo, citando la lungimirante analisi del giornalista e filosofo David Weinberger, il web "è la 
rete più complessa mai creata al mondo: è di molti ordini di grandezza, la più vasta raccolta di scritti e opere dell'uomo di tutta la storia [...]" ma "è stata creata senza l'intervento - singolo - di nessun manager".

Per quanto si cerchi di controllarlo quindi, anche attraverso la progettazione di algoritmi complessi che discriminino perlomeno le informazioni affidabili da quelle non, il web è nato e resta sostanzialmente "un organismo non gestito, al quale non è per ora applicabile un controllo centrale efficiente" (5). Ammesso e non concesso che lo si voglia fare.

Di per sé, questa caratteristica unica della rete non è un difetto, perché internet è solo uno dei mezzi che permettono all'essere umano di fare certe operazioni (6).

Il problema sta nell'interconnessione di questo mezzo con il secondo ingranaggio dell'orologio web: la psicologia umana e i cosiddetti communication bias.

\section{Communication bias ed echo chambers}

Anche nella realtà, la socialità fra individui è favorita quando si hanno interessi, cognizioni, opinioni comuni. Ugualmente avviene nella rete, in maniera amplificata e, utilizzando un'espressione allarmistica, rischiosa (7). Internet permette, ipoteticamente, di creare reti sociali - non a caso si parla di social network - perfettamente personalizzate, prive cioè di quegli elementi di diversità che favoriscono lo scambio di idee e opinioni fra gli appartenenti a una comunità fisica (8).

Nei social, ad esempio, si crea una fortissima omogeneità dei network di amicizie, la quale produce un effetto chiamato "eco nella stanza" (9). È un meccanismo per cui ogni voce all'interno di una rete risulta semplicemente amplificata da chi è d'accordo, facendo perdere progressivamente critiche e dissensi alla discussione (10). In gergo si parla di echo chambers (11).

La formazione di questi gruppi affini avviene per diversi meccanismi automatici propri della rete: gli algoritmi di ricerca di Google e un processo cognitivo detto confirmation bias (12).

Come argomenta Eli Pariser in II filtro. Quello che internet ci nasconde: "gli algoritmi di Google, i misteriosi e affascinanti filtri che selezionano le nostre domande [...] non sono affatto casuali, ma basandosi sulle nostre precedenti richieste (e degli utenti a noi simili) ci guidano verso gusti, opinioni, persone, ambienti, stanze di Weinberger (13) a noi affini, allontanandoci da quelli disomogenei" (14). Ognuno ha il suo personale Google.

Questi modelli matematici di selezione (Google Suggest, il PageRank, Google AdSense, Google Custom Search) agiscono sulla base di un principio: I'informazione generata da un gruppo di persone è in genere sempre più completa di quella prodotta da ogni singolo membro (15). Il PageRank inoltre esclude automaticamente alcuni termini e pagine, perché non sufficientemente utilizzati, perché troppo recenti e/o perché fuori dalle norme specifiche del motore di ricerca (16).

Nessun utente che interagisca, anche minimamente, con la rete, quindi, è immune da questa ghettizzazione delle proprie preferenze.

L'altra determinante delle echo chambers è il confirmation bias. Anche chiamato pregiudizio di conferma, consiste nella tendenza dell'essere umano a selezionare solo le informazioni affini alle proprie convinzioni più profonde e a interpretare le notizie in modo da alimentare i propri preconcetti. È un costrutto psicologico onnipresente in politica, in economia, nella pratica medica e scientifica, e anche nel web (17). Tutto avviene perché ogni individuo ha la tendenza naturale a conservare il proprio sistema di credenze e ad affidarsi ciecamente a esso. È una questione arcaica di sopravvivenza. Ad esempio, quando un soggetto accede a un testo scritto o a un evento porta con sé una serie di "pre-comprensioni" e "pregiudizi" che sono la sua unica vera via per capire. In pratica anche il testo più oggettivo viene interpretato da chi legge. È la Teoria della comprensione di Gadamer (18).

Online, la necessità di autoconfermasi, che come già detto è anche data dalla mancanza di dimensioni fisiche alle quali affidarsi, può essere così forte da portare un utente (o un gruppo) a consumare una bufala anche quando si può facilmente dimostrarne la falsità (19). Se la narrazione di una notizia, anche non vera, si adatta al sistema di valori del lettore, tutto il resto viene semplicemente ignorato.

È un meccanismo che si manifesta, con delle variazioni, anche nella diffusione e citazione di papers scientifici. I dati che non si accordano all'opinione più condivisa, persino se rilasciati da organizzazioni e istituzioni internazionali attendibili, fanno molta più fatica a emergere nei database ufficiali. È il cosiddetto citation bias (20).

Tornando alle echo chambers, le caratteristiche di questi gruppi omogenei vengono amplificate o mitigate anche da altri due elementi specifici delle interazioni di gruppo: I'influenza sociale e la polarizzazione. L'influenza sociale può essere definita come il modo in cui i pensieri, i sentimenti e i comportamenti delle persone vengono influenzati dalla presenza reale o immaginata degli altri. Viene studiata dalla psicologia sociale e agisce con meccanismi estremamente complessi e differenziati dalla situazione. Ha un'enorme rilevanza anche nella determinazione dell'eco nella stanza (21).

La polarizzazione è invece una estremizzazione ingiustificata delle idee, uno dei maggiori rischi delle stanze di Weinberger. Sunstein ha dimostrato che, quando gli appartenenti a una rete sociale online parlano non raggiungono un compromesso, non si muovono verso "il punto intermedio delle tendenze dei membri individuali [...]. I gruppi concludono il processo a una posizione più estrema, nella stessa direzione generale, di quanto non fosse la loro inclinazione all'inizio".

La polarizzazione di un social network (inteso come rete di relazioni) può essere quantificata attraverso una scala che va da 0 a 1. Quando la comunicazione fra echo chambers è nulla il valore è uguale o prossimo all'1. Quando c'è ancora uno scambio tra gruppi, invece, il valore tende a zero.

Una polarizzazione prossima all'uno è caratteristica del 
processo di diffusione massiva delle bufale scientifiche, e soprattutto sanitarie, nei gruppi in rete. Nelle echo chambers di informazione alternativa le notizie si diffondono in maniera lineare. Citando Quattrociocchi e collaboratori: "col crescere del tempo di esposizione alla notizia, cresce il numero di utenti coinvolti. Nelle echo chambers di natura scientifica, invece, c'è un picco iniziale di interesse, durante il quale il numero di utenti coinvolti è molto alto e subito dopo, invece, un calo e standardizzazione". Gli utenti polarizzati su posizioni alternative sono quelli più inclini a diffondere informazioni non verificate; quelle che si diffondono nel web più lentamente ma inesorabilmente.

Insomma le più pericolose.

\section{Il medico e le echo chambers}

Oggi non è raro che molti pazienti arrivino agli ambulatori ben muniti di informazioni fornite dal notissimo "Dr. Google". Non è raro che, proprio a causa di ciò, le indicazioni e le raccomandazioni del medico vengano messe in discussione, tanto che il The Guardian (22) ha definito tutto questo un processo destruente per il rapporto medico paziente.

Molte volte le raccomandazioni del terapeuta vengono non solo rifiutate ma addirittura contraddette in toto con le più varie motivazioni. A questo livello del confronto, indipendentemente dalle affermazioni del medico, il paziente ha già trovato tesi, fatti e documenti che confermano la sua idea online. Capovolgerla diventa così ancora più complesso.

La raccomandazione, in questi casi, è di non entrare mai in competizione con questo tipo di pazienti. Comportamenti efficaci possono essere la mediazione e toni non aggressivi, semmai concilianti.

Prendere di petto soggetti convinti che il riso rosso sia miracoloso non è utile. II rischio è non solo di perderli, ma soprattutto che la persona danneggi se stessa, cosa che un medico ha il dovere di evitare.

Anche i professionisti sanitari non sono comunque immuni dal pregiudizio di conferma. Un esempio è il medico particolarmente sicuro delle proprie diagnosi, fino alla totale svalutazione di altre ipotesi diagnostiche plausibili. Anche in questa situazione, alla stessa stregua del paziente, il medico trova il modo di portare acqua al proprio mulino, selezionando, in maniera principalmente inconsapevole ma effettiva, articoli o revisioni che confermino il suo pensiero.

Il professionista sanitario deve avere sempre presente questo bias cognitivo, che non risparmia, come detto, nessun essere umano. Solo così può saggiamente mettere in discussione il proprio "sapere", accettare il confronto con i colleghi ed essere conscio che la medicina non è una scienza esatta ma probabilistica. Il contraddittorio costruttivo con i colleghi deve essere la prassi.

La diagnosi deve essere basata solo ed esclusivamente sul ragionamento logico, il meno possibile su preconcetti. Solo attraverso uno sforzo del genere può essere costruita una valutazione fondata su prove di efficacia, probabilità e sull'esperienza.

\section{Prospettive per un futuro virtuale}

La necessità della comunicazione sanitaria e scientifica è quella di perturbare questo processo di espansione delle notizie non veritiere, ma ad oggi la ricerca dà risultati sconfortanti sulle sue possibilità.

Alcuni studi hanno dimostrato che una continua esposizione a voci non verificate può essere una utile variabile per l'individuazione di soggetti vulnerabili alla credulità (23).

Colossi digitali come Google e Facebook hanno elaborato e stanno migliorando, con un successo non eccelso in realtà, diverse soluzioni algoritmiche atte a migliorare la visibilità delle news affidabili, a discapito delle bufale (24). II problema è che queste formule matematiche classificano i contenuti solo sulla base di variabili strutturali e, come dimostrato da Walter Quattrociocchi e collaboratori nel 2017, queste non bastano a contrastare la post-verità (25).

Anche il conservatorismo è un problema altrettanto determinante.

"Quasi tutte le informazioni - il 97\% per la precisione tendono a rimanere all'interno della stanza" scelta da ogni utente (26). In parole povere, un dato verificato, che sia di natura medica o di altro tipo, ha solo il $3 \%$ di possibilità di venire recepito da un appartenente a un gruppo esposto a notizie errate, definito, in linguaggio tecnico, un gruppo cospirazionista.

Questi processi, per qualsiasi professionista, non sono certo argomenti di scarso interesse pratico.

In un mondo dove le idee (sotto forma di bit) rappresentano l'unica particella fondamentale costituente le informazioni "reali", diventa essenziale, per lo scienziato, conoscere come le idee stesse si diffondono e vengono preservate.

Per ora, l'unico mezzo utile elaborato per una migliore analisi dei processi che guidano i "communication bias" online e la polarizzazione è un algoritmo matematico appositamente studiato (27).

Agli individui meno coinvolti nella ricerca in questo ambito, ma interessati a contrastare come possibile il processo della mis-informazione, non resta che seguire alcuni consigli pratici, come quelli rilasciati con il Manifesto della comunciazione non ostile (28). Le motivazioni per farsi coinvolgere nella lotta alla disinformazione sono molteplici, ma vengono ben espresse dalle parole di Samantha Power, ambasciatore Usa all'Onu: “È nel nostro interesse coinvolgere le persone che sono più in disaccordo con noi, piuttosto che zittirle. Non soltanto perché ci dà la possibilità di confrontarci con il loro punto di vista, e forse anche cambiarlo, ma soprattutto perché a volte potrebbero aver ragione". 


\section{Disclosures}

Financial support: No financial support was received for this submission.

Conflict of interest: The authors have no conflict of interest.

\section{Bibliografia}

1. Casillo S, Di Trocchio F, Sica S. Falsi giornalistici. Finti scoop e bufale quotidiane. Napoli: Guida Editori. 1997.

2. De Biase L. Facts and cats. For the state of the net. In: Porro $\mathrm{S}$, Molino W. Disinformation technology. Milano: Apogeo 2003:28. http://blog.debiase.com/2016/10/30/facts-cats-statenet-2016/.

3. Castells M. La nascita della società in rete. Milano: Egea SpA. Università Bocconi Editore. 2000, cap. I.

4. Dominici P. La comunicazione nella società ipercomplessa. Condividere la conoscenza per governare il mutamento. Milano: Franco Angeli Editore. 2011:117.

5. Weinberger D. Arcipelago Web. Milano: Sperling \& Kupfer Editori. 2002:36.

6. Ferrarotti F. La perfezione del nulla. Promesse e problemi della rivoluzione digitale. Roma-Bari: Laterza. 1997.

7. Massicci M. La scienza ai tempi dei Social Network. Gli archivi di dati condivisi alla base della collaborazione scientifica online. Trieste: Scuola Superiore di studi avanzati di Trieste. 2016.

8. Wellman B. Physical place and cyberplace: The rise of personalized networking. Int J Urban Reg Res. 2001;25(2):227-52.

9. Del Vicario $M$, Bessi $A$, Zollo $F$, et al. The spreading of misinformation online. Proc Natl Acad Sci. USA. 2016;113(3): 554-9.

10. Sunstein CR. The law of group polarization. J Polit Philos. 2002;10(2):175-95.

11. Quattrociocchi W. Citazione al convegno: Comunicare e promuovere la Salute ai Tempi dei Social Media \#salutesocial, IRCCS - Istituto di Ricerche Farmacologiche Mario Negri, 24 Maggio. 2016.

12. Riotta G. Il web ci rende liberi? Politica e vita quotidiana nel mondo digitale. Einaudi. 2013:17;2.

13. Weinberger D. La stanza intelligente. Torino: Codice Edizioni. 2012:XIV.

14. Pariser E. II filtro. Quello che internet ci nasconde. Milano: II
Saggiatore. 2012.

15. Santoro E, Garattini S. Web 2.0 e social media in medicina: come social network, wiki e blog trasformano la comunicazione, l'assistenza e la formazione in sanità. Roma: II Pensiero Scientifico Editore. 2011:4.

16. Schweiger S, Oeberst A, Cress U. Confirmation bias in webbased search: a randomized online study on the effects of expert information and social tags on information search and evaluation. J Med Internet Res. 2014;16(3):e94.

17. Pines JM. Profiles in patient safety: confirmation bias in emergency medicine. Acad Emerg Med. 2006;13(1):90-4.

18. Festuccia F. L'oggettività dell'informazione: tra mito professionale e ideale regolativo. Roma: Armando Editore. 2010:22.

19. Quattrociocchi W, Vicini A. Misinformation: Guida alla società dell'informazione e della credulità. Milano: Franco Angeli. 2016:74.

20. Greenberg SA. How citation distortions create unfounded authority: analysis of a citation network. BMJ. 2009;339, b2680.

21. Aronson E, Wilson TD, Akert RM. Psicologia sociale. Bologna: II Mulino 2015. A cura di Villano P. 2013.

22. Fagan D. Dr Google knows best: how technology is disrupting our relationships with GPs. The Guardian 27/10/2017. https://www. theguardian.com/technology/2017/oct/28/dr-google-knowsbest-how-technology-is-disrupting-our-relationships-with-gps.

23. Bessi A, Coletto M, Davidescu GA, Scala A, Caldarelli G, Quattrociocchi W. Science vs conspiracy: collective narratives in the age of (mis)information. PloS ONE. 2015;10(2):e0118093.

24. Zollo F, Bessi A, Del Vicario, et al. Debunking in a world of tribes. PloS ONE. 2017;12(7):e0181821.

25. Conti M, Lain D, Lazzeretti R, Lovisotto G, Quattrociocchi W. It's Always April Fools' Day! On the Difficulty of Social Network Misinformation Classification via Propagation Features. 2017; arXiv preprint arXiv:1701.04221.

26. lannaccone S. Bufale: il debunking serve a poco o niente (anzi). 22 aprile 2016. http://www.galileonet.it/2016/04/bufaledebunking-serve-poco-niente/.

27. Del Vicario M, Scala A, Caldarelli G, Stanley HE, Quattrociocchi W. Modeling confirmation bias and polarization. Scientific Reports 7. 2017.

28. Manifesto della comunicazione non ostile. 2017: http://www. paroleostili.com/media-file/manifesto_def.pdf. 